\title{
Near-Edge Absorption Soft X-ray Nanotomography of Cells Incubated with Nanoparticles
}

\author{
José Javier Conesa, ${ }^{1}$ Joaquín Otón, ${ }^{2}$ Eva Pereiro, ${ }^{1}$ Francisco Javier Chichón, ${ }^{2}$ and José L. Carrascosa ${ }^{2,3}$.
}

1. ALBA Synchrotron Light Source. MISTRAL Beamline - Experiments division. Cerdanyola del Vallès, Barcelona, Spain.

2. Centro Nacional de Biotecnología (CNB-CSIC), Cantoblanco, Madrid, Spain.

3. Instituto Madrileño de Estudios Avanzados en Nanociencia (IMDEA Nanociencia), Cantoblanco, Madrid, Spain

Superparamagnetic iron oxide nanoparticles (SPION) have become important tools in nano-biotechnology and nano-biomedicine. These new developments require a precise quantitative analysis at sufficient spatial resolution to model the interactions between nanoparticles and the cellular structures in a quantitative way. The results from this analysis will determine the dose for either drug-delivery or hyperthermia treatments as well as its feasibility. To tackle this issue $15 \mathrm{~nm}$ dimercaptosuccinic acid functionalized SPION were incubated with MCF-7 breast cancer cells [1] as a model system to be analyzed exploiting the iron differential absorption contrast at the L3 iron edge. Near-edge absorption soft X-ray nanotomography (NEASXT) combines whole-cell 3D structure determination at $50 \mathrm{~nm}$ resolution, with 3D elemental distribution and quantification and high throughput. We have solved the three-dimensional distribution and quantification of SPIONs within the cells with sufficient sensitivity to detect the density corresponding to a single nanoparticle in the whole cellular volume (Fig. 1) [2].

References:

[1] M. Chiappi, J.J Conesa, E. Pereiro, J Nanobiotechnology 14(1) (2016), p. 3.

[2] J.J Conesa, J. Otón, M. Chiappi, Scientific Reports 6 (2016), Article number: 22354. 


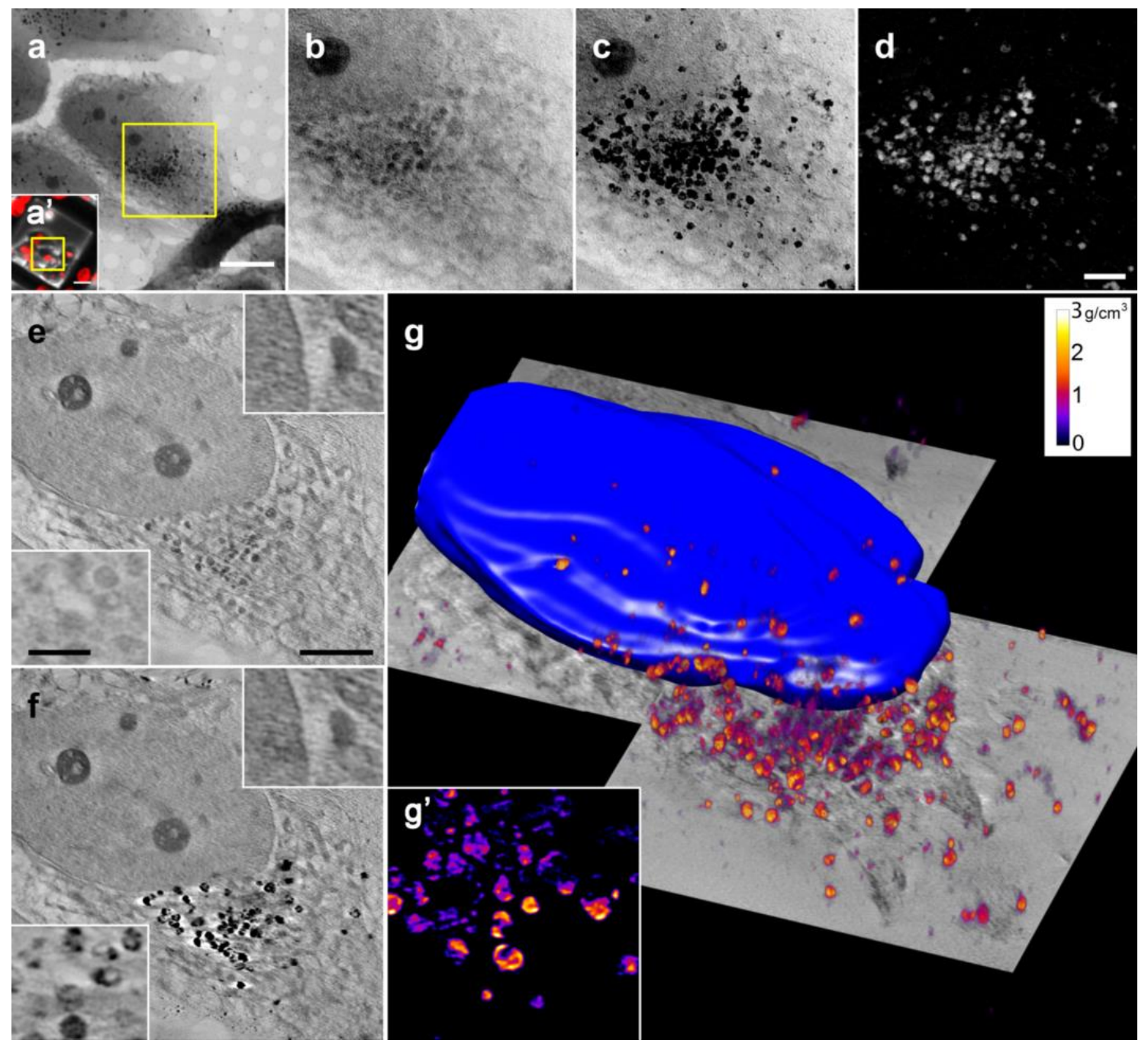

Figure 1. Correlative microscopy, differential tilt-series generation and 3D representation. a) Soft X-ray projection images mosaic (709 eV) of MCF-7 cell incubated with iron nanoparticles. Scale bar $25 \mu \mathrm{m}$. Inset in $\mathrm{a}^{\prime}$ ) In vivo fluorescent image of the same cell. Acidic organelles are labeled with Lysotracker (red). Scale bar, $20 \mu \mathrm{m}$. b) and c) X-ray projection images at 700 and $709 \mathrm{eV}$ respectively. Scale bar, 2 $\mu \mathrm{m}$. d) Difference image generated combining images at 700 and $709 \mathrm{eV}$. Scale bar, 2 $\mu \mathrm{m}$. e) and f) Central slice of reconstructed tomograms at 700 and $709 \mathrm{eV}$ respectively. Scale bar, $2 \mu \mathrm{m}$. g) Volumetric representation of iron oxide densities within the cells, forming clusters near the nucleus. 